\title{
A multi-agent control architecture for a robotic wheelchair
}

doi:10.1533/abbi.2006.0027

\author{
C. Galindo, A. Cruz-Martin, J. L. Blanco, \\ J. A. Fernández-Madrigal and J. Gonzalez \\ E. T. S. I. Informática - Universidad de Málaga, Campus Teatinos, 29071 Málaga, Spain
}

\begin{abstract}
Assistant robots like robotic wheelchairs can perform an effective and valuable work in our daily lives. However, they eventually may need external help from humans in the robot environment (particularly, the driver in the case of a wheelchair) to accomplish safely and efficiently some tricky tasks for the current technology, i.e. opening a locked door, traversing a crowded area, etc. This article proposes a control architecture for assistant robots designed under a multi-agent perspective that facilitates the participation of humans into the robotic system and improves the overall performance of the robot as well as its dependability. Within our design, agents have their own intentions and beliefs, have different abilities (that include algorithmic behaviours and human skills) and also learn autonomously the most convenient method to carry out their actions through reinforcement learning. The proposed architecture is illustrated with a real assistant robot: a robotic wheelchair that provides mobility to impaired or elderly people.
\end{abstract}

Key words: Assistant robots, wheelchair, control architecture, multi-agent.

\section{INTRODUCTION}

The assistant robotics field covers those applications where a mobile robot helps humans to perform certain tasks. Examples of robotic assistant applications are house cleaner and keeper robots, tour guiders, assistant robots for elderly people, etc., in which a robot must work within human environments interacting intelligently with people.

The first consideration to be taken into account in assistant robots is that they are designed to serve non-expert people who usually prefer to communicate and to interact with machines in the same manner they do with other people. Moreover, the presence of a robot within human scenarios like houses, offices, public facilities, etc., imposes a high degree of operation robustness and physical safety for humans as well as a sophisticated set of robot capabilities like manoeuvring within narrow and/or crowded spaces, avoiding mobile obstacles, docking, etc. Planning tasks in such complex and typically large environments

\footnotetext{
Corresponding Author:

C. Galindo

System Engineering and Automation

University of Malaga

Campus Teatinos, 29071

Malaga, Spain

Tel: +34-952132747; Fax: +34-952133361

Email: cipriano@ctima.uma.es
}

also represents a tough problem due to the large number of elements involved.

These issues are usually beyond the capabilities that the current technology offers, so the use of assistant robots operating autonomously within human environments is not yet extended. This lack of robot capabilities could be approached by considering the assisted person (or any other from the surroundings) as an additional component of an augmented robot. That is, humans can be integrated into the robotic system allowing it to extend its abilities through skills either not supported by the robot (i.e. take an elevator) or supported by the robot but in a different and (maybe) more secure manner (i.e. maneuvering in a complex situation). These skills may range from complicated low-level motions to high-level decision makings. Obviously, the robot control architecture must be specifically designed to take into account this degree of interaction.

In the literature, mobile robotic architectures have considered the particular requirements of assistant applications from different perspectives. Some works (Alami et al. 1998; Fernndez-Madrigal and Gonzlez 2002; Fleury et al. 1997) ensure desirable properties as robustness and fault tolerance in the architecture components by providing automatic software design tools, mechanisms to deduce safe actions before they are executed, techniques to check resource availability, etc. In the tele-operation area, whose applications can be seen close to assistant applications, collaborative control (Fong and Thorpe 2002) is used to 
develop robotic architectures that support a tight relation between humans (expert operators) and machines. Through collaborative control, robots accept advice from operators to decide its actions. Such a relation between humans and robots improves the robot-operating capacity, but it restricts the humans to physically act when the robot is not capable to continue its plan, for example, when it passes through a locked door. Other works also consider human-machine integration or cooperation (Fong and Thorpe 2002; Karim 2001; Morris et al. 2002) in a way that it enables a human to provide robot capabilities enough to operate in human environments. In these cases, humans serve only as a command input provider.

Elsewhere a hybrid robotic architecture, called ACHRIN, specifically designed for assistant robots has been presented (Galindo et al. in press; Galindo et al. 2005). ACHRIN facilitates a non-expert person to be involved in the robotics operation when needed, through a special human-robot integration. Such an integration allows the human to participate at all levels, from deliberating a plan to executing it, or even acting as an extra sensor. But in spite of those remarkable features, ACHRIN exhibits some shortcomings:

- Since ACHRIN is a tiered architecture, its components are communicated in a client/server fashion, and thus, communications should be built when designing the architecture. This characteristic prevents the system to be easily scaled up or dynamically modified, i.e. adding components.

- Considering a number of components that perform the same task (redundancy) imposes the necessity of selecting the best one according, for instance, to the experience. In ACHRIN, this selection is carried out by a component that implements a simple and hand-coded policy based on the previous results of execution. However, it would be desirable to adopt a more effective and flexible policy that could provide components with a certain degree of autonomy.

In this article, we propose a multi-agent re-design of ACHRIN to overcome the commented limitations. Multiagent systems (MAS) is a subfield of artificial intelligence (AI) that studies those complex systems formed by several agents that interact in some way. Although MAS is a mature discipline, there is no general agreement on what an agent is, and several definitions can be found in the literature (Franklin and Graesser 1996; Maes 1995; Wooldridge 2002). However, they all have a common aspect: autonomy, learning capabilities and rational behaviour are essential aspects that agents must have. Following this, we have modified ACHRIN by turning all its components into autonomous agents. In our implementation, each agent possesses a mental state; that is, a set of intentions and beliefs, a group of abilities to perform a particular action, i.e. navigation, and a learning mechanism, $Q$-learning (Kaelbling et al. 1996) in our case, to decide at each moment the best ability to accomplish the agent intentions.
MAS becomes a robust and scalable option when managing different elements with different goals that own some kind of information. Moreover, the use of a multi-agent framework seems more appropriate for an assistant robot application than deliberative, reactive or hybrid robotic architectures, since it permits a more natural integration of the human into the robotic system. We refer the reader to Wooldridge (2002) for a complete discussion of MAS.

The rest of this article is organised as follows. Section 'Multi-agent control architecture' gives an overview of the proposed multi-agent architecture. Section 'Inter-agent communication' details inter-agent communications. Section 'Learning capabilities of agents' selects autonomously the most appropriate abilities for performing their actions, and Section 'A real robotic assistant application' describes the application of the multi-agent architecture to a real assistant robot: a robotic wheelchair. Finally, some conclusions and future work are outlined.

\section{MULTI-AGENT CONTROL ARCHITECTURE}

The main feature of the multi-agent architecture presented in this article is the integration of human abilities into the agents that constitute the architecture, as commented further on. In this section, a top-bottom description of the proposed architecture is provided: first, we focus on its structure, that is, the type of agents that includes highlighting the differences with a non-agent-based architecture. Then, we detail the internal structure of each agent, the so-called common agent structure (CAS).

\section{Structure of the multi-agent architecture}

One of the main characteristics that make a multi-agent architecture (as well as any other agent-based system) different from other approaches is the interconnection between their constituent elements. A non-agent-based architecture, as the one shown in Figure 1 (left), establishes a fixed and normally one-to-one or one-to-few-ones connection between their components. In contrast, in a multi-agent approach (Figure 1 right), the agents that make up the robotic architecture are fully interconnected and these connections are completely dynamic.

In particular, our multi-agent architecture, which is an evolution of ACHRIN (Figure 1 left), is called MARCA, * and it is composed of the following agents.

- World Modeller Agent: This agent manages information stemmed from the environment in a human-like manner through the use of a mechanism called abstraction (Galindo et al. in press; Galindo et al. 2005; Kaelbling et al. 1996). It contains both geometric information, i.e. the position of a particular distinctive place and symbolic information, i.e. a human-understandable label to refer to a room.

- Agenda Agent: It accepts and manages requests from different users. Usually people will request the robot to execute

\footnotetext{
*MARCA stands for multi-agent-based robotic control architecture.
} 

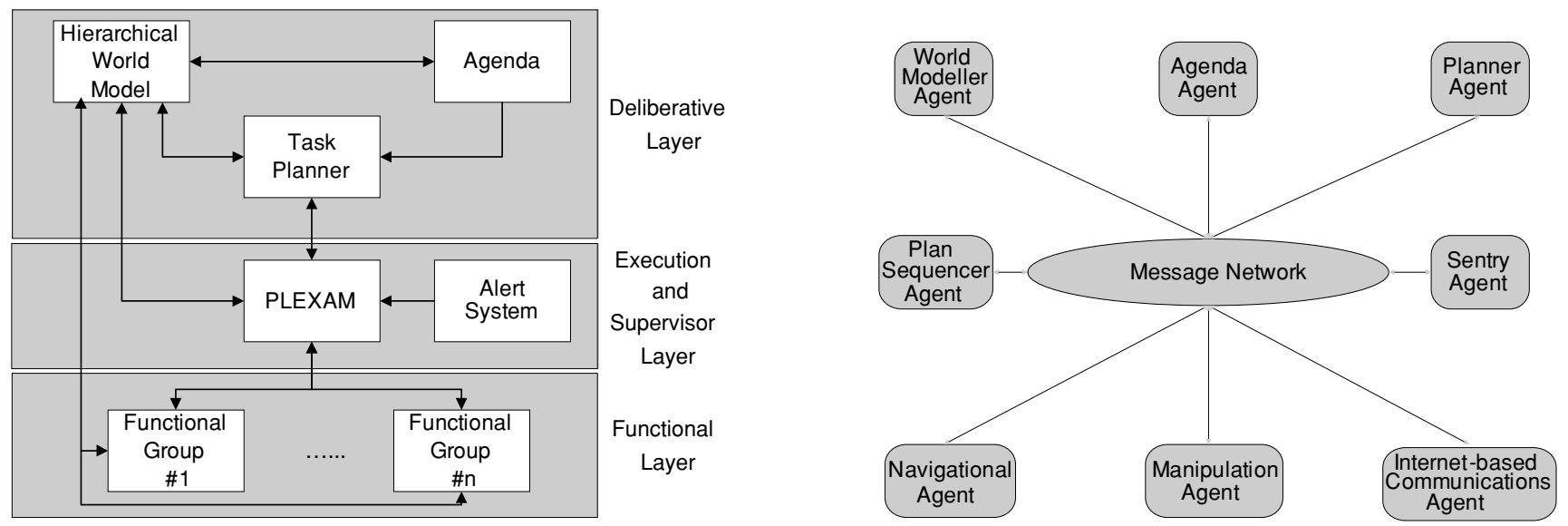

Figure 1 (Left) A scheme of a hybrid robotic architecture (ACHRIN (Galindo et al. in press)). (Right) Its evolution to a multi-agent system. Notice that in a client/server system (left) the addition of a component implies the modification of the components connected to it, which does not occur in a MAS. In our multi-agent re-design of ACHRIN, we have considered navigation, manipulation and Internet-based communication (access to email, for instance) as the functional tasks carried out by the combined human-robot system.

some task, although it is also useful to accept requests from other robots, applications, etc. This enhances the robotic architecture with a higher and more intelligent interaction within its environment. The Agenda broadcasts to the rest of agents the necessity of achieving a goal.

- Planner Agent: This agent is capable of generating a plan to achieve a certain goal taking into account information from the world model as well as the available human + robot abilities. The resultant sequence of abilities that achieves the goal will be taken on by the correspondent agents. For instance, navigation actions will be carried out by the Navigational Agent. In our current implementation, the Planner Agent runs a hierarchical, independent-domain algorithm for planning a variety of tasks (Galindo et al. 2004).

- Sentry Agent: The Sentry Agent is responsible for checking unexpected dangerous situations in the system. It resembles human behaviour since it reacts to external stimuli through both voluntary and reflex actions (Galindo et al. 2004 , in press). A reflex action is an automatic and involuntary movement, which is triggered by an external stimulus, i.e. the 'shut the lids' reflex when an irritating substance enters the eye. On the other hand, a voluntary action is a conscious action, which is carried out in response to a stimulus, for example, we reduce the speed of our vehicle when approaching a red light. Notice that in human beings none of these actions are planned but instinctive. In fact, in neural science the border between reflex and voluntary actions is not well defined (Kuipers 1983). Voluntary and some reflex actions can be learnt and sometimes even inhibited, that is, a person can voluntarily ignore certain stimuli. In this sense, the Sentry Agent can ignore certain stimuli as humans do, in order to adequate its behaviour to the human preferences. For example, a collision stimulus can trigger a stop reflex action during navigation, but maybe the robot is carrying out a shortdistance approach to some object. In such case, the stop reflex can be inhibited to achieve the proposed goal. The major concern when accepting an inhibition is the physical safety of the humans and the robot. Thus, such an inhibition relies on an ad hoc function that takes into account the human preferences, the current action that the robot is executing and the robot state. Other architectures (nonagent-based) also include alert mechanisms (Brugali and Fayad 2002; Fernndez-Madrigal et al. 2004), but none of them provide the capability of consciously ignoring certain alerts from the environment.

- Plan Sequencer Agent: This agent sequences the actions that form a plan and manages the results of their execution. It is in charge of coordinating the different agents that have to perform the actions of the plan. It is also in charge of reacting properly to risky situations, for example, stopping and blocking the vehicle when a collision or a critical battery level alert is reported.

The architecture is completed by a set of agents that physically perform actions. Each agent carries out a particular one, i.e. navigation or manipulation, by possibly using different abilities. For instance, the Navigational Agent can perform navigation through three abilities: a reactive algorithm, a path-planner algorithm and a human manually guiding the vehicle. When an agent accounts for different abilities, it must decide at each moment which is the best one to accomplish its task. This decision process should be autonomous and independently carried out by agents, as we will show in Section 'Learning capabilities of agents'.

In our work we have considered three agents that respectively perform navigation, manipulation and Internetbased communication (i.e. email access). Some results are presented in Section 'A real robotic assistant application'.

\section{Common agent structure}

As it was stated before, the main feature of MARCA is that it distributes some of the abilities of the human among all the agents of the robotic architecture, in order to augment 


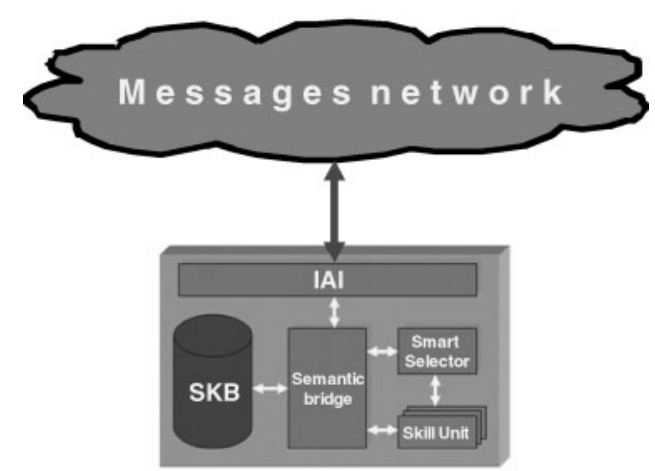

Figure 2 The common agent structure (CAS). All the agents in MARCA are designed using this structure. The number of skill units contained into each agent is variable, and it depends on the agent's functionality as well as on the human and robot capabilities provided by the CAS.

or to improve the functionality of the system. Thus, a human can help the robot to capture world information (as we have demonstrated previously in Fernndez-Madrigal et al. (2004)), or to provide a plan for achieving a goal (interacting with the Planner Agent, as we have shown in Galindo et al. (2004)), or even to physically perform actions like open a door or call an elevator.

This human-robot integration is supported through the use of the CAS as a skeleton for designing every agent (see Figure 2). The CAS enables each agent to consider both human and robot capabilities to accomplish its work through the skill units, previously considered in ACHRIN. Robotic skill units represent abilities of the robot implemented by software algorithms, while human skill units represent the connection of the agent to the human, enabling people to perform actions through appropriate interfaces (i.e. voice communication). An example of the inclusion of human abilities is the case of the Navigational Agent devoted to perform robot navigation: it contains a set of robotic skill units to navigate between two locations, i.e. a reactive navigator and a path-tracker algorithm, but it can also consider the human participation through a human skill unit that permits him or her to manually guide the robot. Thus, the agent accounts for three different ways of accomplishing actions like ' $g$ o to place $X$ '.

One of the commonly accepted features that agents must possess - along with autonomy, proactiveness or communications - is learning. So, among the different possibilities that an agent accounts for accomplishing its tasks, it should be able to learn over time the most convenient one, given its experience and the current conditions of the environment (Murch and Johnson 1999). Within the CAS skeleton, the Smart Selector (SS) is in charge of the agent's learning process, and it is always active to adapt the agent performance with respect to variable environmental conditions, as commented in more detail in Section 'Learning capabilities of agents'.
All skill units considered by the SS to accomplish a requested action pursue the same goal (i.e. to reach to a destination in the case of navigating), but they may exhibit differences in the way they are invoked, especially in the case of human units. For instance, the navigation action 'go to $\mathrm{pl}^{\text {'* }}$ can be carried out by invoking a reactive navigation algorithm (a robotic skill unit) in the form 'navigate to $(x=$ $0.2, y=12.3, \phi=12$ )', while the human skill unit would require a linguistic label that represents the destination in terms of the human knowledge, i.e. go to Peter's office.

The translation into the skill unit parameters from an action requested by any other agent in the architecture is carried out by the Semantic Bridge (SB). This component also permits the internals of the agent (Smart Selector and skill units) to request/provide data to other agents of the architecture (i.e. it deals directly with the semantics explained in Section 'Inter-agent communication'). For instance, the previously commented human skill unit can request an external agent for the linguistic label of the destination.

The SB is also in charge of maintaining the Semantic Knomledge Base (SKB) that represents the internal mental state of the agent in terms of intentions and beliefs. Such an internal mental state is updated by the SB with incoming messages or requests from other agents. Communications between agents rely on the Inter-Agent Interface (IAI) that provides communication primitives to send/receive messages following a fixed semantic, as commented in more detail in Section 'Inter-agent communication'.

\section{INTER-AGENT COMMUNICATION}

In this section we focus on how agents communicate with each other. Since agents are autonomous entities with their own motivations, they cannot be forced by other agents to do some action; hence, communication is used to influence or persuade them to perform tasks.

In contrast with traditional software architectures, in which communication between different pieces of software usually makes intensive use of the client-server paradigm (just using procedural calls, as in our previous architecture ACHRIN, RPC or any other function invocation mechanism), MAS imposes a much richer set of interactions between agents. Thus, if communications are expected to affect the internal mental states of agents, they should not be mere raw data, but information about agent attitudes.

In our approach, inter-agent communications are based on message passing, which is the most common scheme for MAS. In particular, we use the standardised message format proposed by FIPA, namely ACL (Foundation for Intelligent Physical Agents 2002b). We have been highly inspired by the CAL specification (Foundation for

\footnotetext{
* $p 1$ is a symbol stored in the internal world model of the robot that represents a particular location of its workspace. Symbols are managed by planner agents to plan robot tasks.
} 
Table 1 The communication acts that we use in our system to define the semantics of inter-agent communications*

\begin{tabular}{|c|c|c|c|c|}
\hline \multirow{2}{*}{$\begin{array}{l}\text { Performatives } \\
\text { (sent from } i \text { to } j \text { ) }\end{array}$} & \multicolumn{3}{|c|}{ Sender (Agent $i$ ) } & \multirow{2}{*}{$\begin{array}{l}\text { Receiver (Agent } j \text { ) } \\
\text { Effects in SKB }\end{array}$} \\
\hline & Trigger & Preconditions & Effects in SKB & \\
\hline $\operatorname{inform}(j, \phi)$ & $+\mathrm{D}_{i} \mathrm{~B}_{j} \phi$ & $\mathrm{B}_{i} \phi$ & $-\mathrm{D}_{i} \mathrm{~B}_{j} \phi$ & $+\mathrm{B}_{j} \phi$ \\
\hline request $(j, a)$ & $+\mathrm{D}_{i}$ Done $(a)$ & $\begin{array}{l}\neg \mathrm{B}_{i} \text { Agent }(i, a) \wedge \\
\mathrm{B}_{i} \text { Agent }(j, a) \wedge \\
\text { Preconditions }(a) \wedge \\
\neg \mathrm{B}_{i} \mathrm{~B}_{j} \mathrm{D}_{i} \text { Done }(a)\end{array}$ & $+\mathrm{B}_{i} \mathrm{~B}_{j} \mathrm{D}_{i}$ Done $(a)$ & $\begin{array}{l}+\mathrm{B}_{j} \mathrm{D}_{i} \text { Done }(a) \\
\text { Accept: }+\mathrm{I}_{j} \text { Done }(a) \\
\text { Reject: }+\mathrm{D}_{j} \mathrm{~B}_{i} \neg \mathrm{I}_{j} \text { Done }(a)\end{array}$ \\
\hline $\operatorname{agree}(j, a)$ & $+\mathrm{I}_{i}$ Done $(a)$ & $\begin{array}{l}\mathrm{B}_{i} \mathrm{D}_{j} \text { Done }(a) \wedge \\
\neg \mathrm{B}_{i} \mathrm{~B}_{j} \mathrm{I}_{i} \text { Done }(a)\end{array}$ & $+\mathrm{B}_{i} \mathrm{~B}_{j} \mathrm{I}_{i}$ Done $(a)$ & $\begin{array}{l}+\mathrm{B}_{j} \mathrm{I}_{i} \text { Done }(a) \\
+\mathrm{B}_{j} \text { Feasible }(a)\end{array}$ \\
\hline refuse $(j, a)$ & $+\mathrm{D}_{i} \mathrm{~B}_{j} \neg \mathrm{I}_{i}$ Done $(a)$ & $\begin{array}{l}\mathrm{B}_{i} \mathrm{D}_{j} \text { Done }(a) \wedge \\
\neg \mathrm{B}_{i} \mathrm{~B}_{j} \neg \mathrm{I}_{i} \text { Done }(a)\end{array}$ & $+\mathrm{B}_{i} \mathrm{~B}_{j} \neg \mathrm{I}_{i}$ Done $(a)$ & $+\mathrm{B}_{j} \neg \mathrm{I}_{i}$ Done $(a)$ \\
\hline $\operatorname{cancel}(j, a)$ & $-\mathrm{D}_{i}$ Done $(a)$ & $\begin{array}{l}\mathrm{B}_{i} \mathrm{~B}_{j} \mathrm{I}_{i} \text { Done }(a) \wedge \\
\neg \text { Done }(a)\end{array}$ & $-\mathrm{B}_{i} \mathrm{~B}_{j} \mathrm{I}_{i}$ Done $(a)$ & $\begin{array}{l}-\mathrm{B}_{j} \mathrm{D}_{i} \text { Done }(a) \\
\text { And probably: } \\
-\mathrm{I}_{j} \text { Done }(a)\end{array}$ \\
\hline failure $(j, a)$ & $-\mathrm{I}_{i}$ Done $(a)$ & $\begin{array}{l}\mathrm{B}_{i} \mathrm{~B}_{j} \mathrm{I}_{i} \text { Done }(a) \wedge \\
\neg \text { Done }(a)\end{array}$ & $-\mathrm{B}_{i} \mathrm{~B}_{j} \mathrm{I}_{i}$ Done $(a)$ & $\begin{array}{l}+\mathrm{B}_{j} \neg \mathrm{I}_{i} \text { Done }(a) \\
+\mathrm{B}_{j} \neg \text { Feasible }(a)\end{array}$ \\
\hline query $(j, \beta)$ & $+\mathrm{D}_{i}$ Done $(\operatorname{Eval}(\beta))$ & $\begin{array}{l}\neg \mathrm{B}_{i} \operatorname{Agent}(\mathrm{i}, \operatorname{Eval}(\beta)) \wedge \\
\mathrm{B}_{i} \operatorname{Agent}(j, \operatorname{Eval}(\beta))\end{array}$ & - & $+\mathrm{D}_{j} \mathrm{~B}_{i} \operatorname{Eval}(\beta)$ \\
\hline
\end{tabular}

${ }^{*}$ The nomenclature is explained in detail in the text.

Intelligent Physical Agents 2002a) to define the underlying semantics of the communicative acts.

Mental attitudes of agents in our architecture are defined using these operators:

- Beliefs: The operator $B_{i} p$ means that agent $i$ believes that fact $p$ holds.

- Desires: The operator $D_{i} a$ means that action $a$ is a goal to be achieved by agent $i$.

- Intentions: The operator $I_{i} a$ means that agent $i$ is intent on carrying out the action $a$. The semantic meaning of intention differs from the concept of goal normally used in robotics; intentions are used in MAS for invoking the execution of actions.

Moreover, to model actions, we need the following additional operators:

- Agent $(i, a)$ : Means that agent $i$ is capable of performing the action $a$.

- Done (a): Represents that action $a$ has been carried out.

- Feasible (a): This means that, for a given instant of time, preconditions for executing action $a$ are met.

The set of communicative acts or performatives that we use in MARCA and their associate preconditions, triggers and effects is detailed in Table 1 . There we have used the prefixes $+/-$ to designate the insertion/removal of facts from the agents SKB. Since our agent execution model is event driven, these modifications in an agent's mental state trigger actions into the IAI and the Semantic Bridge, for instance, the execution of another communicative act or the initiation of a given skill unit.

The meaning of the specified performatives is explained as follows:
- Inform: Implies the intention of an agent $i$ of letting another agent $j$ to know something that $i$ currently believes. We assume a sincere behaviour of agents; that is, the content communicated by an agent represents its current attitudes, which are immediately incorporated into the beliefs of the receiver agent.

- Request: Sender agent requests the receiver to execute a given action $a$. The target agent has to decide whether to accept or refuse the proposal. As shown in Table 1, the SKB of the target agent is consequently updated to reflect the new intention of performing the action (in case of acceptance), or the desire of communicating the rejection to the sender agent in other case.

- Agree: The agreement of an agent $i$ to perform a requested action for some other agent $j$. This communicative act is triggered on acceptation for a request.

- Refuse: Agent $i$ refuses the request of agent $j$ for performing the action $a$.

- Cancel: Informs to an agent $j$ that agent $i$ has no longer the intention of agent $j$ of performing the action $a$. As a consequence, the receiver agent will leave its intention of carrying out the action if there is no other agent that still desires its execution.

- Failure: Agent $i$ aims to perform action $a$ for another agent $j$, but it was not possible to complete the execution and currently the agent does no longer intent on trying it.

- Query: This performative contains an expression $\beta$, whose meaning is not standardised, but it is assumed that the receiver agent should be able to evaluate it. In the preconditions of the performative, queries are sent only when the agent cannot solve the expression, thus communications are used only when the agent needs external information.

To pass messages between agents, the performatives are sent as formatted strings, which are coded as an ACL 


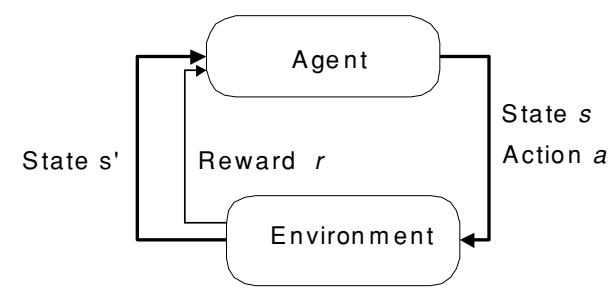

Figure 3 The Reinforcement Learning framework implemented in our CAS Smart Selector. Agents learn their skill unit selection policy from their interaction with the environment. The execution of a certain action (skill unit) produces a reward value that guides the learning process over time.

compliant plain text message. In Section 'A real robotic assistant application', we will show examples of such messages.

\section{LEARNING CAPABILITIES OF AGENTS}

One of the main characteristics that define an agent is its capability for learning from its own experience. In MARCA, agents must learn how to select the appropriate skill unit (either human or robotic) when carrying out their actions. For that purpose, we have chosen Reinforcement Learning (RL) to be implemented in our CAS Smart Selector, since RL has a thoroughly studied formal foundation and it also seems to obtain good results when applied to mobile robotics tasks (Liu and Wu 2001; Smart and Kaelbling 2002).

RL has been analysed widely elsewhere; the classical survey by Kaelbling et al. (1996) is a good starting point for a deeper research into the subject (Sutton and Barto 1998). In short, RL is a machine-learning paradigm where an agent in a state $s$ executes some action $a$ turning its state into $s^{\prime}$ and getting a reinforcement signal or reward $r$. Those experience tuples $\left(s, a, s^{\prime}, r\right)$ are used for finding a policy $\pi$ that maximises some long-run measure of reward. It is supposed that the environment where the agent evolves is a non-deterministic one, which means that taking the same action in the same state on different occasions may yield different next states and/or rewards. RL is graphically explained in Figure 3.

There is a comprehensive bunch of methodologies for modelling and solving RL problems. A well-known solution is Markov Decision Processes (MDPs), which can be defined by

- a set of states $S$,

- a set of actions $A$,

- a reward or reinforcement function $R: S \times A \rightarrow \mathfrak{R}$ meaning that if the agent is in state $s$ and performs action $a$, it receives an $r$ reinforcement signal, and

- a state transition function $T: S \times A \rightarrow \Pi(s)$, where $\Pi(s)$ is a probability distribution over the set $S$. So, probability of making a transition from current state $s$ to next state $s^{\prime}$ executing action $a$ is written as $T\left(s, a, s^{\prime}\right)$.
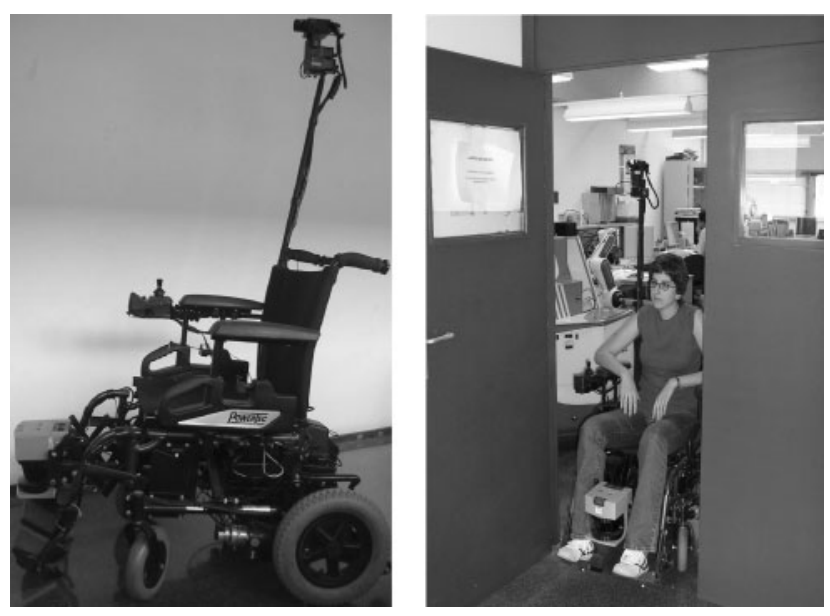

Figure 4 Two views of the SENA robotic wheelchair in which we have evaluated our human-robot integration architecture.

Upon these sets and functions, an optimal policy $\pi$ that maximises the obtained reward can be computed in this way:

- The optimal value of a state $V^{*}(s)$ is the expected reward that the agent will gain if it starts in that state and executes the optimal policy. This optimal value is stated as the sum of the expected reward $R$ plus the expected discounted value of the next state, using the best available action:

$$
\begin{gathered}
V^{*}(s)=\max _{a}\left(R(s, a)+\gamma \sum_{s^{\prime} \in S} T\left(s, a, s^{\prime}\right) V^{*}\left(s^{\prime}\right)\right), \\
\forall s \in S
\end{gathered}
$$

where parameter $\gamma$ is a discount factor that represents how much attention is paid to future rewards.

- The optimal policy then is specified by the expression:

$$
\pi^{*}(s)=\underset{a}{\arg \max }\left(R(s, a)+\gamma \sum_{s^{\prime} \in S} T\left(s, a, s^{\prime}\right) V^{*}\left(s^{\prime}\right)\right) .
$$

Both reinforcement and state transition functions are named a model. However, such a model is not always known in advance; in fact, most of robotics applications cannot provide that prior knowledge. In these situations, there is an approach that can be used for learning the optimal policy: Q-learning. Q-learning uses the following optimal value function $Q^{*}$ :

$$
Q^{*}(s, a)=R(s, a)+\gamma \sum_{s^{\prime} \in S} T\left(s, a, s^{\prime}\right) \max _{a^{\prime}} Q^{*}\left(s^{\prime}, a^{\prime}\right)
$$

Since the $Q$-function makes the action explicit, it can be recursively computed on line by means of

$$
Q(s, a)=Q(s, a)+\alpha\left(r+\gamma \max _{a^{\prime}} Q\left(s^{\prime}, a^{\prime}\right)-Q(s, a)\right)
$$




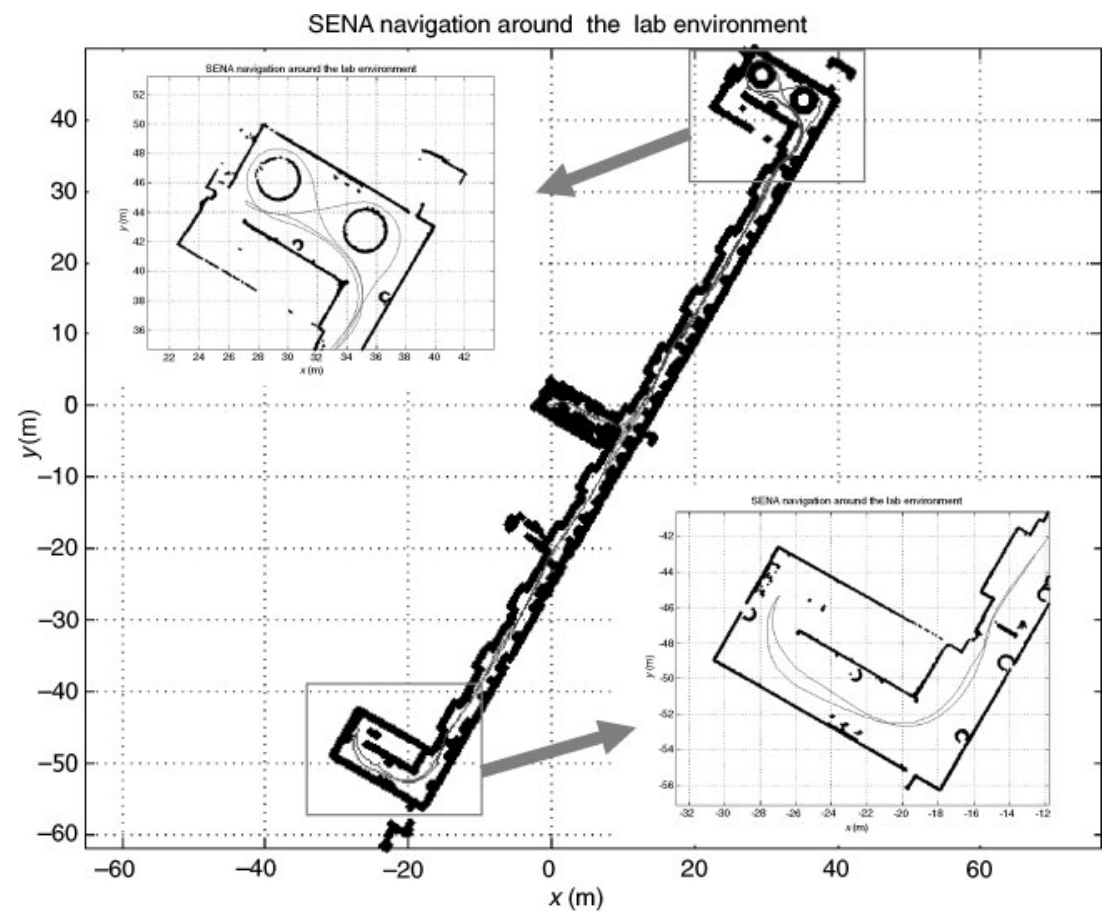

Figure 5 An example of SENA navigation in the surroundings of our laboratory. Rooms and corridors are depicted in thick lines, whereas the path followed by SENA is shown in thin lines. For illustrating the variety and difficulty of the paths performed by SENA in this environment, two interesting zones have been zoomed.

Parameter $\alpha$ is the learning rate, and it must slowly decrease to guarantee convergence of function $Q$ (Even-Dar and Mansour 2003). Once the optimal $Q$-function $Q^{*}$ is obtained, the optimal policy can be fixed as it was stated in (2).

For illustrating how $Q$-learning performs the learning process, we focus our attention on the Navigational Agent. This agent is in charge of the mobility issues of the robot: it must provide a safe displacement of the vehicle in its environment as it reaches some given spatial references. As it was previously commented, there are three skill units available in the NA: a human skill unit that puts humans in charge of movement through a joystick, a path-tracker algorithm that follows a previously calculated path, and a robotic reactive skill unit that implements a reactive navigation algorithm (Blanco et al. 2005). The agent learns via $Q$-learning an optimal policy that states which skill unit is the best navigation option at every moment.

The mathematical formulation of the $Q$-learning technique for the NA is as follows.

1. Every state is built as the sum of five factors, namely: unexpected obstacles detection $(\mathrm{O})$, local minimum detection (LM), critical obstacles detection - doors, ramps, etc., that demand human intervention - (CO), available robot energy (RE) and human energy (HE). Both obstacle detection $(\mathrm{O}$ and $\mathrm{CO})$ can be implemented by means of laser range sensors. The presence of a local minimum (LM) is detected when the robot is not able to leave a section of the route for a certain time. This is a typical drawback of reactive navigation (Ulrich and Borenstein 1998). Finally, energy levels are measured in terms of battery levels (RE) and human fatigue (HE), respectively; human fatigue is due to the effort done by the human since he or she has to be alert during the navigation in order to avoid any hazardous unexpected situation. This fatigue can be measured through the frequency of actuations on the joystick and the frequency of alerts produced by the human within the Sentry Agent. The first three factors (O, LM and CO) have been discretised into yes/no values, whereas energy factors (RE and HE) have been discretised into low/medium/high values. The result of this discretisation is a set of 72 possible states in the evolution of the navigational system.

2. The set of actions $A$ simply matches the set of skill units of the agent, since the policy-to-learn looks for the best sequence of skill units to apply. In this way, the possible actions are HUMAN, DELIBERATIVE and REACTIVE navigation.

3. The reinforcement function $R$ favours states with a high level of available energy along with actions that can be applied with a low human-robot interaction effort. In this way, a HUMAN action requires a high-interaction effort since it needs to activate the voice communication with the human; the REACTIVE action does not need any interaction and the DELIBERATIVE action would be an in-between that just demands some kind of path/speed planification. The final goal of this definition of reward is to obtain policies with an energetic and interaction effort as low as possible. 


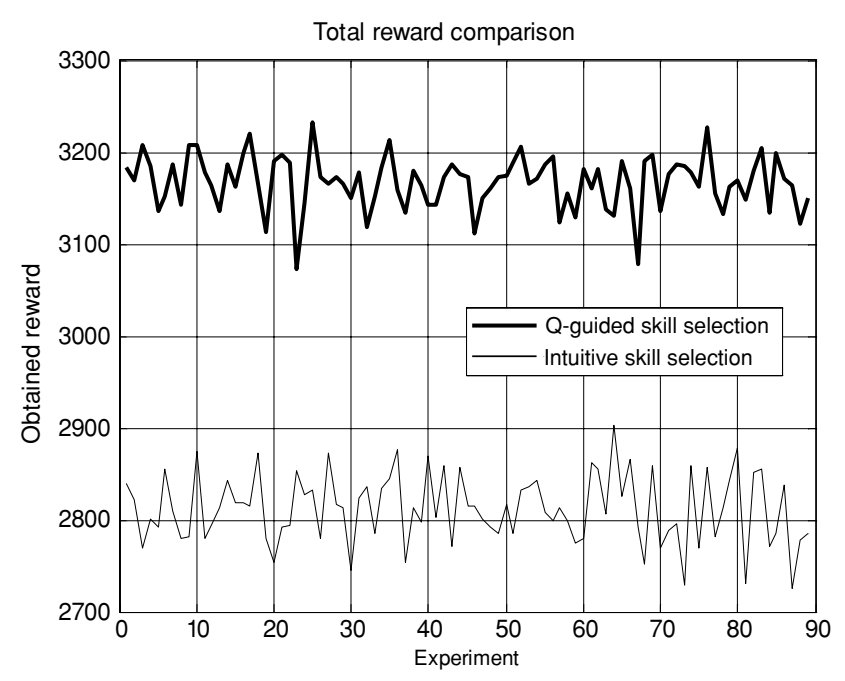

Figure 6 Q-learning (solid line) versus intuitive algorithm (dashed line) rewards. The policy found by $Q$-learning offers better results than using an intutive algorithm.

In the following section, the results of some experiments conducted on the Navigational Agent for evaluating our RL approach are shown.

\section{A REAL ROBOTIC ASSISTANT APPLICATION}

MARCA has been tested on an assistant robot called SENA (see Figure 4). It is a robotic wheelchair based on a commercial powered wheelchair that has been equipped with several sensors and an onboard computer to reliably perform high-level tasks in indoor environments. SENA accounts for Wi-Fi connection capabilities that improve to a great extent the possibilities of our robot, ranging from tele-operation of the vehicle to the human driver access to the Internet.
Our tests have been carried out within our laboratory and near corridors, in which the user can select a destination via voice. Since the main operation of SENA is navigation, we have focussed our experiences on the Navigational Agent; an example of a navigation task around our laboratory environment can be seen in Figure 5.

In a navigation task, the Planner Agent interacts with the user (as described in Galindo et al. (2004)) for constructing the best route to the goal, and then sends it to the NA via the Messages Network, using the semantics described in Section 'Inter-agent communication'. When a navigation step arrives at the agent, its SB translates it to the Smart Selector to perform the action. Then, the Smart Selector applies the $Q$-learning algorithm, and the best skill unit learnt until that moment will be activated.

Our results reveal that $Q$-learning is effectively working and provides an important degree of autonomy in the selection of each agent's abilities, producing good policies for navigational issues. We have compared the reward obtained by $Q$-learning against the reward obtained by an intuitive algorithm that selects a supposed best skill unit, implemented through a common-sense approach. Basically, the intuitive algorithm selects HUMAN action whenever a critical obstacle comes up; it selects DELIBERATIVE action if neither unexpected obstacles nor local minima are found, and, finally, uses REACTIVE action in every other situation. The comparison is plotted in Figure 6.

As it can be seen, $Q$-learning finds a policy of actions that gets better rewards than the intuitive algorithm: average rewards are 3167 ( $Q$ ) and 2812 (int), and standard deviations are 29.4 and 37.9, respectively, which makes $Q$-learning an outstandingly better option.

Furthermore, $Q$-function really converges to a certain value after a proper number of learning steps. Figure 7 shows, for two states (expressed as a combination of the five factors mentioned in Section 'Learning capabilities of agents'), how $Q$-function of every action (HUMAN,
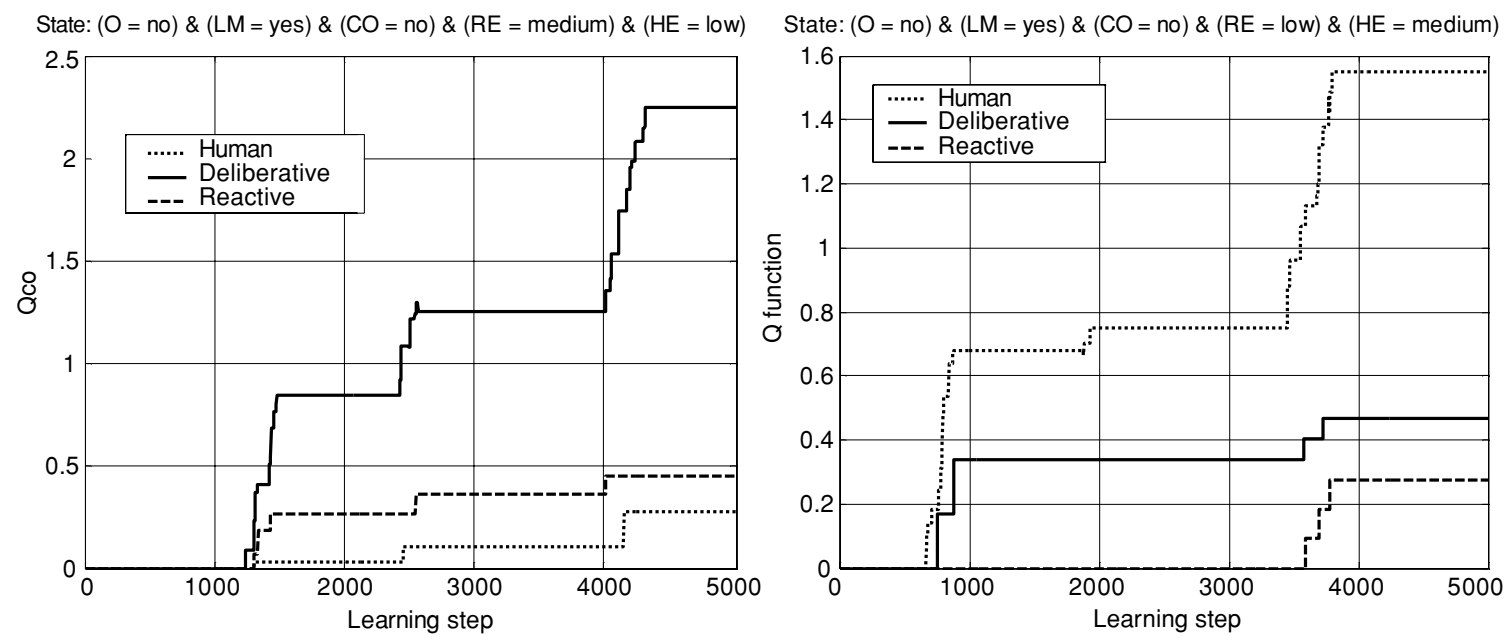

Figure 7 Q-function values for two selected states. The selected best actions are DELIBERATIVE and HUMAN actions, respectively. 

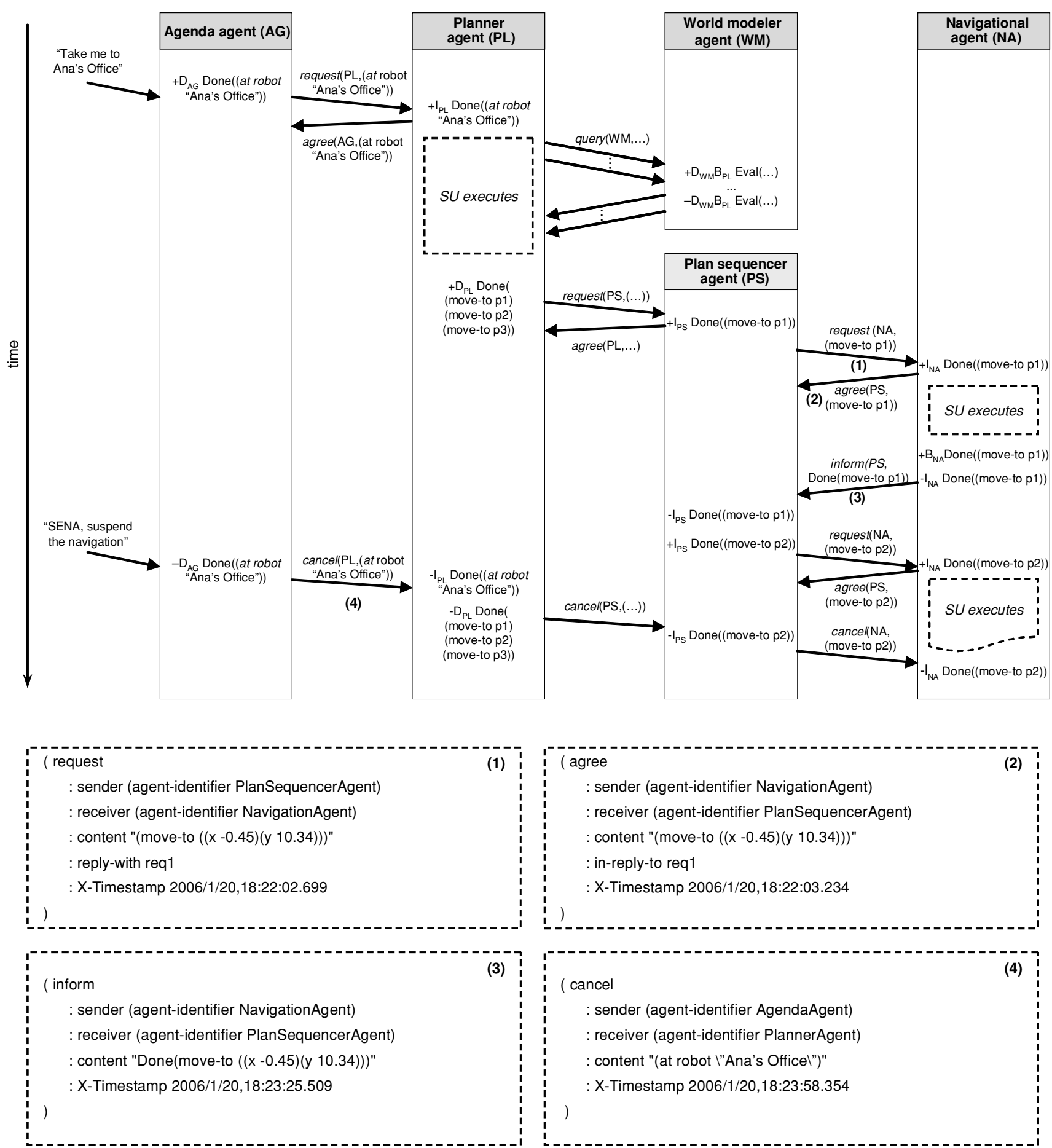

Figure 8 The schematic representation of agents' internal states and some messages sent during the experiment described in the text.

DELIBERATIVE and REACTIVE) finally converges. It can be seen that, although both states are similar in terms of unexpected obstacles, local minima and critical obstacles, their different energy levels lead to a different skill unit selection.

Regarding the internal state of agents and how communicative acts are used between them, please refer to Figure 8 , in which the main changes in agents' mental state are shown to illustrate the evolution of their attitudes through an experiment with SENA. Some messages corresponding to key communicative acts are also sketched as arrows between agents. We can interpret the semantic meaning of the agents attitudes as follows. At first, the user enters a navigation command to the robot via voice, which is captured by an SU inside the Agenda Agent as a new desire: $D_{\mathrm{AG}}$ Done ((at robot 'Ana's Office')). This complex 
task must be decomposed by the Planner Agent, thus the user command is requested to this agent, which agrees and introduces into its mental state the intention of carrying out this action. This event triggers the execution of an internal SU, which queries the World Modeller Agent the abstract information needed to plan the complex action. Once that a plan consisting of elementary actions is available, a new desire in the Planner Agent's SKB for executing the plan leads to a request to the Plan Sequencer Agent, which also agrees and incorporates the plan to its intentions. This agent therefore sends subsequent requests to the Navigational Agent, whose SS will select the most promising SU for performing the demanded navigation at each instant of time through the previous $Q$-leaning approach. In this experiment, the user asks the robot to abort the navigation while the second elementary action is being executed. As can be seen in Figure 8, the user annulment of the navigation task produces a chain of cancel messages between agents, as well as the retreat of intentions from their SKB. As a result, all the involved agents return to their previous states and the navigation action becomes definitively forgotten at all the abstraction levels, from high-level pathplanning to low-level local navigation algorithms. Finally, we should highlight that messages between agents have been implemented as ACL plain-text messages, as can be seen with the messages denoted as (1)-(4) in Figure 8. We use the standard fields and an additional timestamp field, which can be employed to measure communication delays, among other possible uses.

\section{CONCLUSIONS AND FUTURE WORK}

Robots that operate in human environments, especially in assistant robotic applications, may allow the researchers to relax somehow the typical autonomy requirements of a conventional robotic platform. However, this leads to a greater amount of work on human-robot interaction issues. Commonly, human-robot interaction is considered as just a simple communication between the robot architecture and the human. However, we believe that assistant robotics needs a much stronger interaction if the robot has to face successfully complex situations, especially those that it cannot solve by its own means. Thus, the human must be integrated into the robotic architecture as a part of an augmented system, in order to achieve the so-called human-robot integration that we claim in our work.

In this article, we have presented MARCA, a multiagent robotic architecture that enables such human-robot integration. We have chosen a multi-agent system approach rather than other conventional architectures because agents are closer to represent the human as a part of the system than other software constructions (modules, procedures, objects, etc.). Agents, as well as humans, have intentions and mental states, and use some semantics in their communications. In addition, they have some learning capabilities that allow them to adapt to environmental changes and to achieve good performances over time. Furthermore, MAS systems also offer other valuable benefits, like robustness or scalability.

We have defined the semantics for the agents to communicate and maintain their internal mental states, as well as how the semantics are translated into practical requests for the algorithms of the architecture. Besides, since any agent in MARCA is endowed with a set of robotic and/or human skill units for performing some action, it must decide which of them is the best in every situation. We have implemented a $Q$-learning procedure that learns this association over time, trying to optimise the long-term behaviour of the system.

MARCA has been applied to a real robotic wheelchair, and the suitability and effectiveness of the architecture to this kind of application has been experimentally validated.

This is not a finished work, and these preliminary results form the basis of an ongoing work. In fact, this article is a first step towards the inclusion of the human as an agent within the architecture, since at this stage the human is not yet an agent, but his or her capabilities are distributed inside any agent that needs them for augmenting its skills. Furthermore, we continue with our work with assistant robots (such as our robotic wheelchair SENA, or our recent tour guider robot SANCHO) to implement solutions for important requirements of assistant applications.

\section{ACKNOWLEDGMENT}

This work was supported by the Spanish Government under research contract CICYT05-01391.

\section{REFERENCES}

Alami R, Chatila R, Fleury S, et al. 1998. An architecture for autonomy. Int $\mathcal{F}$ Robotics Res. Special issue on Integrated Architectures for Robot Control and Programming, 17(4):315-37.

Blanco J L, González J, Fernández-Madrigal JA. 2005. The PT-Space: A new Space Representation for Non-Holonomic Mobile Robot reactive Navigation. Technical Report, University of Málaga.

Brugali D, Fayad ME. 2002. Distributed computing in robotics and automation. IEEE Trans Robotics Automation, 18(4):409-20.

Cohen PR, Levesque HJ. 1990. Intention is choice with commitment. Art Intellig, 42(3).

Even-Dar E, Mansour Y. 2003. Learning rates for $Q$-learning. 7 Mach Learn Res, 5:1-25.

Fernández JL, Simmons RG. 1998. Robust execution monitoring for navigation plans. In Proceedings of the IEEE / RSJ International Conference on Intelligent Robots and Systems, Victoria, British Columbia, Canada.

Fernández-Madrigal JA, González J. 2002. A visual tool for robot programming. In 15th IFAC World Congress on Automatic Control, Barcelona, Spain.

Fernández-Madrigal JA, González J. 2002. Multihierarchical Graph Search. IEEE Trans PAMI, 24(1).

Fernández-Madrigal JA, González J. 2001. Multi-Hierarchical Representation of Large-Scale Space. International Series on 
Microprocessor-Based and Intelligent Systems Engineering. Vol. 24. Kluwer Academic Publishers, Netherlands.

Fernández-Madrigal JA, Galindo C, González J. 2004. Assistive navigation of a robotic wheelchair using a multihierarchical model of the environment. Integrated Computer-Aided Eng, 11:309-322.

Fleury S, Herrb M, Chatila R. GenoM: A tool for the specification and the implementation of operating modules in distributed robot architecture. In IEEE/RSJ International Conference on Intelligent Robots and Systems (IROS'97), Grenoble, France.

Fong TW, Thorpe C. 2002. Robot as partner: Vehicle teleoperation with collaborative control. In Proceedings of the 2002 NRL Workshop on Multi-Robot Systems.

Foundation for Intelligent Physical Agents. 2002a. ACL Communicative Act Library Specification. URL: http://www.fipa.org. Document identifier SC00037.

Foundation for Intelligent Physical Agents. 2002b. ACL Message Structure Specification. URL: http://www.fipa.org. Document identifier SC00061.

Franklin S, Graesser A. 1996. Is it an agent, or just a program?: A taxonomy for autonomous agents. In Proceedings of the 3rd International Workshop on Agent Theories, Architectures and Languages. Springer-Verlag.

Galindo C, González J, Fernández-Madrigal JA. A Control Architecture for Human-Robot Integration: Application to a Robotic Wheelchair. IEEE Systems, Man and Cybernetics Part B, 36(5):1053-67.

Galindo C, González J, Fernández-Madrigal JA. 2005. An architecture for close human-robot interaction. Application to rehabilitation robotics. In IEEE International Conference on Mechatronics and Automation (ICMA'2005), Ontario (Canada), July-August 2005.

Galindo C, Fernández-Madrigal JA, González J. 2004. Hierarchical task planning through world abstraction. IEEE Trans. Robotics, 20(4):667-690.

Galindo C, González J, Fernández JA. 2004. Interactive task planning through múltiple abstraction: Application to assistant robotics. In 16th European Conference on Artificial Intelligence, Valencia, Spain, August 2004.

Harnand S. 1987. Psychological and cognitive aspects of categorical perception: A critical overview. In Harnan S, ed. Categorical Perception: The Groundwork of Cognition. New York: Cambridge University Press, Chapter 1.

Hirtle SC, Jonides J. 1985. Evidence of hierarchies in cognitive maps. Memory Cogn, 13(3).

Kaelbling LP, Littman ML, Moore AW. 1996. Reinforcement learning: A survey. 7 Artif Intell Res, 4:237-277.

Kandel E, Schwartz JH. 1985. Principles of Neural Science. Elsevier Science Publishing Co. Inc., Chapter 33.
Karim T. 2001. A semi-autonomous reactive control architecture. 7 Intell Robotic Syst, 32.

Khatib O. 2002. Human-centered robotics and haptic interaction: From assistance to surgery, the emerging applications. In 3rd International Workshop on Robot Motion and Control.

Kuipers BJ. 1983. The cognitive map: Could it have been any other way? In Picks HL, Acredolo LP, eds. Spatial Orientation: Theory, Research and Applications. New York: Plenum Press, pp. 345-359.

Liu J, Wu J. 2001. Multi-Agent Robotic Systems. CRC Press.

Maes P. 1995. Modeling adaptative autonomous agents. In Langton CG, ed. Artificial Life: An Overview. Cambridge, MA: The MIT Press, pp. 135-162.

Morioka K, Lee JH, Hashimoto H. Human centered robotics in intelligent space. In Proceedings of the IEEE ICRA'02, Washington, DC.

Morris AC, Smart CK, Thayer SM. 2002. Adaptative multi-robot multi-operator work systems. In Proceedings of the 2002 NRL Workshop on Multi-Robot Systems.

Murch R, Johnson R. 1999. Intelligent Software Agents. Prentice-Hall.

Prochazka A, Clarac F, Loeb GE, et al. 1999. What Do Reflex and Voluntary Mean? Modern Views on an Ancient Debate. Springer-Verlag.

Remolina E, Fernández JA, Kuipers BJ, et al. 1999. Formalizing regions in the spatial semantic hierarchy: An AH-graphs implementation approach. Lecture Notes Comput Sci, 1661:109-124.

Scholtz J. 2003. Theory and evaluation of human-robot interaction. In Proceedings of the 36th International Conference on System Sciences, Hawai.

Smart WD, Kaelbling LP. 2002. Effective reinforcement learning for mobile robots. In International Conference on Robotics and Automation, Washington, DC.

Sutton RS, Barto AG. 1998. Reinforcement Learning: An Introduction. Cambridge, MA: The MIT Press.

Tahboub KA. 2001. A semi-autonomous reactive control architecture. F Intell Robotics Syst, 32(4):445-459.

Ulrich I, Borenstein J. 1998. VFH+: Reliable obstacle avoidance for fast mobile Robots. In Proceedings of IEEE International Conference on Robotics and Automation.

Veloso M, Stone P. 2002. Robot teams: From diversity to polymorphism. In A Survey of Multiagent and Multirobot Systems, pp. 37-92. Balch T, Parker LE, eds. Wellesley, MA: AK Peters.

Wooldridge M. 2002. An Introduction to MultiAgent Systems. John Wiley \& Sons Ltd. 

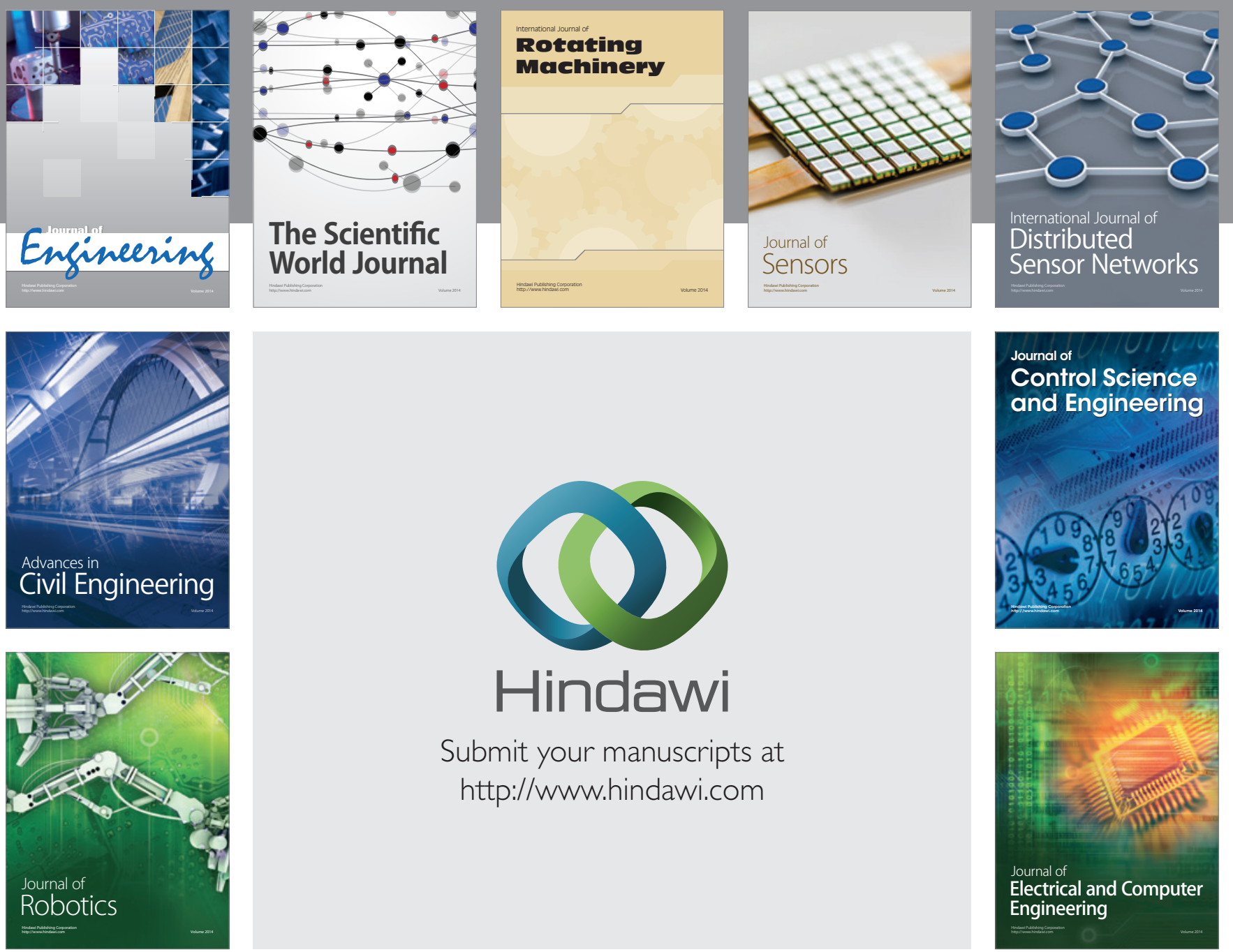

Submit your manuscripts at

http://www.hindawi.com
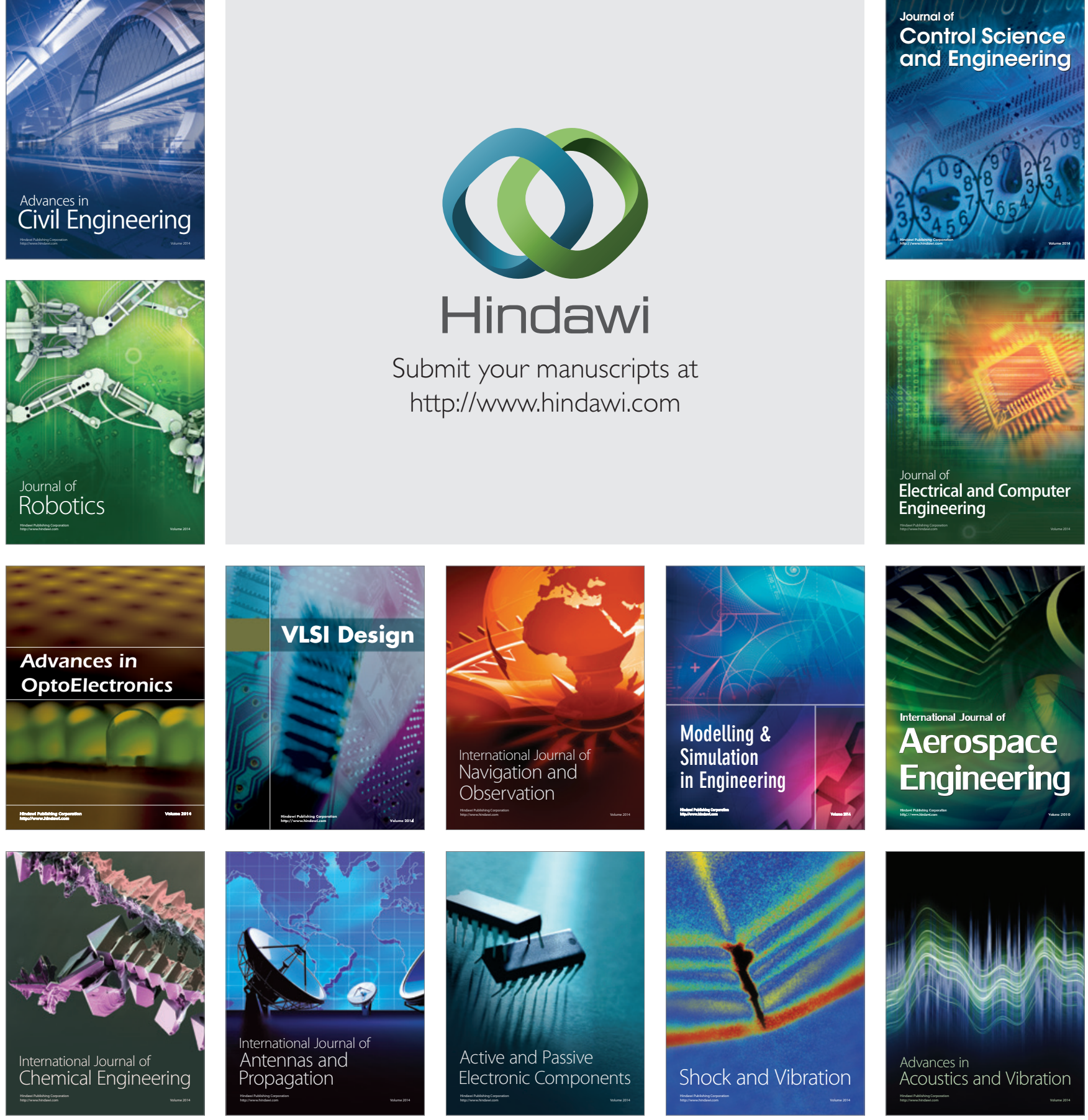